\title{
Outcome Prediction after Neoadjuvant Chemotherapy (NAC) for Breast Cancer, using Tumor-Infiltrating Lymphocytes within Fibrotic Foci of Tumor Stroma (FF- TILs)
}

\section{Yuka Asano}

Osaka City University Graduate School of Medicine

\section{Shinichiro Kashiwagi ( $\nabla$ spqv9ke9@view.ocn.ne.jp)}

Osaka City University Graduate School of Medicine https://orcid.org/0000-0002-0460-9599

Rika Kouhashi

Osaka City University Graduate School of Medicine

\section{Akimichi Yabumoto}

Osaka City University Graduate School of Medicine

\section{Koji Takada}

Osaka City University Graduate School of Medicine

\section{Sae Ishihara}

Osaka City University Graduate School of Medicine

\section{Wataru Goto}

Osaka City University Graduate School of Medicine

\section{Tamami Morisaki}

Osaka City University Graduate School of Medicine

Masatsune Shibutani

Osaka City University Graduate School of Medicine

\section{Hiroaki Tanaka}

Osaka City University Graduate School of Medicine

Kosei Hirakawa

Osaka City University Graduate School of Medicine Masaichi Ohira

Osaka City University Graduate School of Medicine

\section{Research}

Keywords: tumor-infiltrating lymphocytes, neoadjuvant chemotherapy, breast cancer, fibrotic foci, prognostic marker

Posted Date: January 21st, 2021

DOI: https://doi.org/10.21203/rs.3.rs-150877/v1 
License: @ (i) This work is licensed under a Creative Commons Attribution 4.0 International License. Read Full License 


\section{Abstract}

Background: Tumor-infiltrating lymphocytes (TILs), which are indicators for monitoring an immune response, are generally mononuclear immunocytes that aggregate with tumors and are thought to have a close relationship with cancer cells. On the other hand, a fibrotic focus (FF) within the stroma of a tumor is a histological formation that plays an important role in the cancer microenvironment with regard to proliferation and development. Here, we focus on TILs that exist within the FF and we have performed pathological evaluations.

Methods: Of the 320 patients were treated with neoadjuvant chemotherapy (NAC), 239 subjects who were able to evaluate FF-TILs were targeted. Lymphocytes that infiltrate the FF are FF-TILs.

Results: The disease-free survival (DFS) period after NAC for the high-FF-TIL group was found to be significantly longer than that for the low-FF-TIL group for all cases $(p<0.001)$ and for all subtypes of triple-negative breast cancer (TNBC) $(p=0.001)$, human epidermal growth factor receptor 2-enriched breast cancer (HER2BC) $(p=$ $0.010)$, and hormone receptor-positive breast cancer (HRBC) $(p=0.003)$. In multivariable analysis as well, highFF-TIL group classification was an independent factor for recurrence after NAC for all cases $(p<0.001$, hazard ratio $(H R)=0.198)$ and all subtypes of TNBC $(p=0.006, H R=0.172)$, HER2BC $(p=0.025, H R=0.135)$, and $\operatorname{HRBC}(p=0.007, \mathrm{HR}=0.228)$.

Conclusions: It is suggested that FF-TILs are a useful factor for predicting recurrence of breast cancer after NAC.

Article headings: NAC for breast cancer by evaluation of FF-TILs

\section{Background}

Cancer cells have various mutations that allow them to proliferate spontaneously and survive. However, the surrounding environment also influences cancer cells in their entirety and their intrinsic characteristics [1]. Therefore, monitoring the host immune response to tumors in the cancer microenvironment helps predict treatment response and outcome [1, 2]. Tumor-infiltrating lymphocytes (TILs), which are indicators for monitoring an immune response, are generally mononuclear immunocytes that aggregate with tumors and are thought to have a close relationship with cancer cells. Recently, the usefulness of methods that histopathologically evaluate the TILs function in situ has been elucidated [3-5]. Moreover, it has been shown that morphological evaluation of TILs is useful for outcome prediction in the treatment of breast cancer, as well as for predicting the therapeutic effects of drugs; therefore, TILs are considered novel biomarkers [6-9].

Methods for in situ evaluation of TILs and their cutoff values have not assimilated previously, but rather have depended on the previous reports $[7,10,11]$. Thus, in 2014, a recommendation concerning evaluation methods for TILs was created by the International Working Group [3]. TILs can be classified on the basis of the region in which they exist as either stromal TILs (within the stroma of the tumor) or intratumoral TILs (existing within tumor foci and therefore in contact with cancer cells). According to this recommendation, it is desirable to evaluate TILs according to the quantity of immune cells within the cancer stroma; therefore, this recommendation employs evaluation with stromal TILs. However, it has been suggested that the heterogeneity of TILs deters clear cutoff values from being defined. 
Recently, it has been reported that the biological state of the cancer stroma is closely related to cancer proliferation and development [12-14]. Therefore, the evaluation of histomorphological images of tumor stroma has come to be considered one of the major keys to understanding the cancer microenvironment. A fibrotic focus (FF) within the stroma of a tumor is a histological formation that plays an important role in the cancer microenvironment with regard to proliferation and development $[15,16]$. Infiltrating tumor cells surround the FF, which can be recognized as a converged focus of the tissue component that exists within the tumor. In breast cancer, it has been reported that tumors with wide-ranging FF have a high likelihood of malignancy and poor prognosis $[15,17,18]$. That is, it is thought that morphological evaluation of FF is an important indicator in the cancer microenvironment for understanding the transformation to malignancy $[19,20]$.

Here, we focus on TILs that exist within the FF and we have performed pathological evaluations. TILs within FF are called FF-TILs, and we hypothesized that the evaluation of TILs that exist within these regions would be a more precise indicator than previous methods of evaluating TILs. Among patients undergoing neoadjuvant (presurgical) chemotherapy (NAC) for breast cancer, we evaluated the prediction of treatment effects using FF-TILs.

\section{Methods}

\section{Patient background}

A total of 320 patients with resectable, early-stage breast cancer diagnosed as stage IIA (T1, N1, M0 or T2, N0, $\mathrm{M0})$, IIB (T2, N1, M0 or T3, N0, M0), or IIIA (T1-2, N2, M0 or T3, N1-2, M0) were treated with NAC between 2005 and 2015. The present study included 239 participants, following the exclusion of 24 patients in whom the evaluation of TILs from the biopsy samples was difficult, and another 57 patients in whom the evaluation of FFTILs was difficult (Fig. 1). Tumor stage and $\mathrm{T}$ and $\mathrm{N}$ factors were stratified on the basis of the TNM Classification of Malignant Tumors, Union for International Cancer Control (UICC) Seventh Edition [21]. Breast cancer was confirmed histologically by core needle biopsy (CNB) or vacuum-assisted biopsy (VAB), and staged by systemic imaging studies using computed tomography (CT), ultrasonography (US), and bone scintigraphy. Breast cancer was classified into subtypes according to the immunohistochemical expression of estrogen receptor (ER), progesterone receptor (PgR), human epidermal growth factor receptor (HER) 2, and Ki67. Based on their immunohistochemical expression profiles, the tumors were categorized into the following immunophenotypes: luminal A (ER + and/or PgR+, HER2-, Ki67-low); luminal B (ER + and/or PgR+, HER2+) (ER + and/or PgR+, HER2-, Ki67-high); HER2-enriched (HER2BC) (ER-, PgR-, and HER2+); and triple-negative breast cancer (TNBC) (negative for ER, PgR and HER2). In this study, luminal A and luminal B were considered to be hormone receptor-positive breast cancer (HRBC) [22].

All patients received a standardized protocol of NAC consisting of four courses of FEC100 $\left(500 \mathrm{mg} / \mathrm{m}^{2}\right.$ fluorouracil, $100 \mathrm{mg} / \mathrm{m}^{2}$ epirubicin, and $500 \mathrm{mg} / \mathrm{m}^{2}$ cyclophosphamide) every 3 weeks, followed by 12 courses of $80 \mathrm{mg} / \mathrm{m}^{2}$ paclitaxel administered weekly. The patients with HER2-positive breast cancer, were additionally administered weekly $(2 \mathrm{mg} / \mathrm{kg})$ or tri-weekly $(6 \mathrm{mg} / \mathrm{kg})$ trastuzumab during paclitaxel treatment [23-25]. All patients underwent chemotherapy as outpatients. Therapeutic anti-tumor effects were assessed in accordance with the Response Evaluation Criteria in Solid Tumors (RECIST) criteria [26]. Patients underwent mastectomy or breast-conserving surgery following NAC [27]. The pathological effect of chemotherapy was assessed for resected primary tumors after NAC. Pathological complete response (pCR) was defined as the complete 
disappearance of the invasive components of the lesion with or without intraductal components, including that in the lymph nodes according to the National Surgical Adjuvant Breast and Bowel Project (NSABP) B-18 protocol [28]. All patients who underwent breast-conserving surgery were administered postoperative radiotherapy to the remnant breast. The standard postoperative adjuvant therapy for the subtype concerned was administered.

Overall survival (OS) time was the period from the initiation of NAC to the time of death from any cause. Disease-free survival (DFS) was defined as freedom from all local, locoregional, and distant recurrences. All patients received follow-up with physical examination every 3 months, US every 6 months, and CT and bone scintigraphy annually. The median follow-up period was 3.7 years (range, $0.2-6.0$ years) for the assessment of OS and 3.4 years (range, $0.1-6.0$ years) for DFS.

\section{Ethics}

This study was conducted at Osaka City University Graduate School of Medicine, Osaka, Japan, according to the Reporting Recommendations for Tumour Marker prognostic Studies (REMARK) guidelines and a retrospectively written research, pathological evaluation, and statistical plan [29]. Written informed consent was obtained from all patients. This research conformed to the provisions of the Declaration of Helsinki in 2013. The study protocol was approved by the Ethics Committee of Osaka City University (\#926).

\section{Histopathological Evaluation Of Tils}

Histopathological assessment of predictive factors was made for CNB or VAB specimens at the time of the breast cancer diagnosis. Histopathological parameters examined included nuclear grade, histological type, presence of TILs, and correlation of these parameters with intrinsic subtypes and PCR. Histopathological analysis of the percentage of TILs was evaluated on a single full-face hematoxylin and eosin (HE)-stained tumor section according to criteria described by Salgado et al [3]. TILs were defined as the infiltrating lymphocytes within tumor stroma and were expressed by the proportion to the field investigated, and the number of TILs in stroma surrounding the stained cancer cells was quantitatively measured in each field under 400-times magnification $[11,30]$. The area of in situ carcinoma and crush artifacts were not included. Proportional scores were defined as $3,2,1$, and 0 if the area of stroma with lymphoplasmacytic infiltration around invasive tumor cell nests was $>50 \%,>10-50 \%, \leq 10 \%$, and absent, respectively (Fig. 2A-D). TILs were considered high when scores were $\geq 2$ and low when scores were 1 and 0 .

\section{Histopathological evaluation of FF-TILs}

An FF is a scar-like lesion consisting of an area of mainly collagen and fibroblasts, often located near the center of a carcinoma. And, an FF is a converged focus of the tissue component of the stroma of a tumor, and it is surrounded by infiltrating tumor cells. An FF is defined as "FF often consisted of fibrous bands radially expanding to the surrounding area, and FF was located within the tumor, was surrounded by a more cellular zone of infiltrating ductal carcinoma cells, and occupied various percentage of the tumor area" $[15,16]$. The fibroblasts and collagen fiber that form the FF show a storiform pattern, and propagate intertwined with each other. Lymphocytes that infiltrate the FF are FFTILs. We refer to the high TIL group (TIL score $\geq 2$ ) within an FF as the high-FF-TIL group, and the low TIL group (TIL score: 1 or 0 ) within a FF as the low-FF-TIL group. We refer 
to the prediction of treatment effect using previous TIL evaluation methods as the Training Set (TS), and that using FF-TILs as the Validation Set (VS). Histopathologic evaluation of TILs and FF was jointly performed by two breast pathologists (MO, YK) who were blinded to clinical information, including treatment allocation and outcomes.

\section{Sectional analysis}

Statistical analysis was performed using the SPSS version 19.0 statistical software package (IBM Corp., Armonk, NY). The associations between FF-TILs, and clinicopathological variables were examined using the chisquare test (or Fisher's exact test when necessary). Multivariable analysis of pCR was carried out using a binary logistic regression model. The Kaplan-Meier method was used to estimate DFS and OS, and the results were compared between groups with log-rank tests. The Cox proportional hazards model was used to compute univariable and multivariable hazard ratios (HR) for the study parameters with $95 \%$ confidence intervals (Cls), and used in a backward stepwise method for variable selection in multivariable analyses. A p value $<0.05$ was considered significant. Cutoff values for different biomarkers included in this study were chosen before statistical analysis.

\section{Results}

\section{FF-TILs and clinicopathological investigation}

Among the 239 patients who underwent NAC, 123 (51.5\%) were in the high-FF-TIL group, and 116 (48.5\%) were in the low-FF-TIL group. In the high-FF-TIL group, the Ki67 value was significantly high ( $p=0.049)$, the proportion of HRBC was significantly low $(p=0.013)$, and the $p C R$ rate was significantly high $(p=0.004)$ (Table 1). With regard to subtypes, there were 83 cases of TNBC (34.7\%), 46 cases of HER2BC (19.2\%), and 110 cases of HRBC (46.1\%). In an investigation that considered the clinicopathological background, regarding HRBC, in the high-FFTIL group the Ki67 values were significantly high $(p=0.001)$ (Table 2$)$. However, for all the subtypes, no correlation with the pCR rate was found (TNBC: $p=0.154$, HER2BC: $p=0.489$, HRBC: $p=0.083$ ). 
Table 1

Correlation between clinicopathological features and FF-TILs in 239 breast cancers.

\begin{tabular}{|c|c|c|c|}
\hline \multirow[t]{2}{*}{ Parameters } & \multicolumn{2}{|r|}{ FF-TILs in all breast cancers $(n=239)$} & \multirow[t]{2}{*}{$\mathrm{p}$ value } \\
\hline & High $(n=123)$ & Low $(n=116)$ & \\
\hline Age at operation & $60(48.8 \%)$ & $56(48.3 \%)$ & 0.938 \\
\hline$\leq 55$ & $63(51.2 \%)$ & $60(51.7 \%)$ & \\
\hline \multicolumn{4}{|l|}{$>55$} \\
\hline Menopause & $52(42.3 \%)$ & $46(39.7 \%)$ & 0.680 \\
\hline Negative & $71(57.7 \%)$ & $70(60.3 \%)$ & \\
\hline \multicolumn{4}{|l|}{ Positive } \\
\hline Tumor size & $23(18.7 \%)$ & $15(12.9 \%)$ & 0.223 \\
\hline$\leq 2 \mathrm{~cm}$ & $100(81.3 \%)$ & $101(87.1 \%)$ & \\
\hline \multicolumn{4}{|l|}{$>2 \mathrm{~cm}$} \\
\hline Lymph node status & $36(29.3 \%)$ & $30(25.9 \%)$ & 0.556 \\
\hline Negative & 87 (70.7\%) & $86(74.1 \%)$ & \\
\hline \multicolumn{4}{|l|}{ Positive } \\
\hline Nuclear grade & $97(78.9 \%)$ & $92(79.3 \%)$ & 0.932 \\
\hline 1,2 & $26(21.1 \%)$ & $24(20.7 \%)$ & \\
\hline \multicolumn{4}{|l|}{3} \\
\hline Ki67 & $47(38.2 \%)$ & $59(50.9 \%)$ & 0.049 \\
\hline$\leq 14 \%$ & $76(61.8 \%)$ & $57(49.1 \%)$ & \\
\hline \multicolumn{4}{|l|}{$>14 \%$} \\
\hline Intrinsic subtype & $47(38.2 \%)$ & $36(31.0 \%)$ & 0.244 \\
\hline TNBC & $76(61.8 \%)$ & $80(69.0 \%)$ & \\
\hline \multicolumn{4}{|l|}{ non-TNBC } \\
\hline Intrinsic subtype & $29(23.6 \%)$ & $17(14.7 \%)$ & 0.080 \\
\hline HER2BC & 94 (76.4\%) & 99 (85.3\%) & \\
\hline non- HER2BC & & & \\
\hline
\end{tabular}

FF, fibrotic focus. TILs, tumor-infiltrating lymphocytes. TNBC, triple-negative breast cancer. HER2BC, human epidermal growth factor receptor 2-enriched breast cancer. HRBC, hormone receptor-positive breast cancer. $\mathrm{pCR}$, pathological complete response. 


\begin{tabular}{|c|c|c|c|}
\hline \multirow[t]{2}{*}{ Parameters } & \multicolumn{2}{|r|}{ FF-TILs in all breast cancers $(n=239)$} & \multirow[t]{2}{*}{$\mathrm{p}$ value } \\
\hline & High $(n=123)$ & Low $(n=116)$ & \\
\hline Intrinsic subtype & $47(38.2 \%)$ & $63(54.3 \%)$ & \multirow[t]{2}{*}{0.013} \\
\hline $\begin{array}{l}\text { HRBC } \\
\text { non-HRBC }\end{array}$ & $76(61.8 \%)$ & $53(45.7 \%)$ & \\
\hline Pathological response & $51(41.5 \%)$ & $28(24.1 \%)$ & \multirow[t]{2}{*}{0.004} \\
\hline $\begin{array}{l}\text { pCR } \\
\text { non-pCR }\end{array}$ & $72(58.5 \%)$ & $88(75.9 \%)$ & \\
\hline $\begin{array}{l}\text { FF, fibrotic focus. TILs, } \\
\text { epidermal growth facto } \\
\text { pCR, pathological comp }\end{array}$ & $\begin{array}{l}\text {-infiltrating lymphoc } \\
\text { tor 2-enriched brea } \\
\text { esponse. }\end{array}$ & $\begin{array}{l}\text { TNBC, triple-negative breast cancer. } \\
\text { ancer. HRBC, hormone receptor-positiv }\end{array}$ & $\begin{array}{l}\text { human } \\
\text { cancer. }\end{array}$ \\
\hline
\end{tabular}


Table 2

Correlations between FF-TILs and clinicopathological parameters in 83 triple-negative, 46 HER2 enriched, and 110 luminal type breast cancers.

\begin{tabular}{|c|c|c|c|c|c|c|c|c|c|}
\hline \multirow[t]{2}{*}{ Parameters } & \multicolumn{2}{|c|}{ TNBC $(n=83)$} & \multirow{2}{*}{$\begin{array}{l}p \\
\text { value }\end{array}$} & \multicolumn{2}{|c|}{ HER2BC $(n=46)$} & \multirow{2}{*}{$\begin{array}{l}p \\
\text { value }\end{array}$} & \multicolumn{2}{|c|}{ HRBC $(n=110)$} & \multirow{2}{*}{$\begin{array}{l}\mathrm{p} \\
\text { value }\end{array}$} \\
\hline & $\begin{array}{l}\text { High } \\
(n= \\
47)\end{array}$ & $\begin{array}{l}\text { Low }(n= \\
36)\end{array}$ & & $\begin{array}{l}\text { High }(n \\
=29)\end{array}$ & $\begin{array}{l}\text { Low }(n \\
=17)\end{array}$ & & $\begin{array}{l}\text { High }(n \\
=47)\end{array}$ & $\begin{array}{l}\text { Low }(n \\
=63)\end{array}$ & \\
\hline $\begin{array}{l}\text { Age at } \\
\text { operation }\end{array}$ & $\begin{array}{l}25 \\
(53.2 \%)\end{array}$ & $\begin{array}{l}19 \\
(52.8 \%)\end{array}$ & 0.970 & $\begin{array}{l}14 \\
(48.3 \%)\end{array}$ & $\begin{array}{l}7 \\
(41.1 \%)\end{array}$ & 0.641 & $\begin{array}{l}24 \\
(51.1 \%)\end{array}$ & $\begin{array}{l}34 \\
(54.0 \%)\end{array}$ & 0.763 \\
\hline $\begin{array}{l}\leq 55 \\
>55\end{array}$ & $\begin{array}{l}22 \\
(46.8 \%)\end{array}$ & $\begin{array}{l}17 \\
(47.2 \%)\end{array}$ & & $\begin{array}{l}15 \\
(51.7 \%)\end{array}$ & $\begin{array}{l}10 \\
(58.9 \%)\end{array}$ & & $\begin{array}{l}23 \\
(48.9 \%)\end{array}$ & $\begin{array}{l}29 \\
(46.0 \%)\end{array}$ & \\
\hline & $\begin{array}{l}19 \\
(40.4 \%)\end{array}$ & $\begin{array}{l}14 \\
(38.9 \%)\end{array}$ & 0.887 & $\begin{array}{l}11 \\
(37.9 \%)\end{array}$ & $\begin{array}{l}7 \\
(41.1 \%)\end{array}$ & 0.828 & $\begin{array}{l}22 \\
(46.8 \%)\end{array}$ & $\begin{array}{l}25 \\
(39.7 \%)\end{array}$ & 0.455 \\
\hline Positive & $\begin{array}{l}28 \\
(59.6 \%)\end{array}$ & $\begin{array}{l}22 \\
(61.1 \%)\end{array}$ & & $\begin{array}{l}18 \\
(62.1 \%)\end{array}$ & $\begin{array}{l}10 \\
(58.9 \%)\end{array}$ & & $\begin{array}{l}25 \\
(53.2 \%)\end{array}$ & $\begin{array}{l}38 \\
(60.3 \%)\end{array}$ & \\
\hline & $\begin{array}{l}7 \\
(14.9 \%)\end{array}$ & & 0.897 & $\begin{array}{l}7 \\
(24.1 \%)\end{array}$ & $\begin{array}{l}2 \\
(11.8 \%)\end{array}$ & 0.268 & $\begin{array}{l}9 \\
(19.1 \%)\end{array}$ & $\begin{array}{l}8 \\
(12.7 \%)\end{array}$ & 0.355 \\
\hline $\begin{array}{l}\leq 2 \mathrm{~cm} \\
>2 \mathrm{~cm}\end{array}$ & $\begin{array}{l}40 \\
(85.1 \%)\end{array}$ & $\begin{array}{l}31 \\
(86.1 \%)\end{array}$ & & $\begin{array}{l}22 \\
(75.9 \%)\end{array}$ & $\begin{array}{l}15 \\
(88.2 \%)\end{array}$ & & $\begin{array}{l}38 \\
(80.9 \%)\end{array}$ & $\begin{array}{l}55 \\
(87.3 \%)\end{array}$ & \\
\hline $\begin{array}{l}\text { Lymph node } \\
\text { status }\end{array}$ & $\begin{array}{l}14 \\
(29.8 \%)\end{array}$ & $\begin{array}{l}9 \\
(25.0 \%)\end{array}$ & 0.629 & $\begin{array}{l}10 \\
(34.4 \%)\end{array}$ & $\begin{array}{l}5 \\
(29.4 \%)\end{array}$ & 0.723 & $\begin{array}{l}12 \\
(25.5 \%)\end{array}$ & $\begin{array}{l}16 \\
(25.4 \%)\end{array}$ & 0.987 \\
\hline $\begin{array}{l}\text { Negative } \\
\text { Positive }\end{array}$ & $\begin{array}{l}33 \\
(70.2 \%)\end{array}$ & $\begin{array}{l}27 \\
(75.0 \%)\end{array}$ & & $\begin{array}{l}19 \\
(65.6 \%)\end{array}$ & $\begin{array}{l}12 \\
(70.6 \%)\end{array}$ & & $\begin{array}{l}35 \\
(74.5 \%)\end{array}$ & $\begin{array}{l}47 \\
(74.6 \%)\end{array}$ & \\
\hline $\begin{array}{l}\text { Nuclear } \\
\text { grade }\end{array}$ & $\begin{array}{l}36 \\
(76.6 \%)\end{array}$ & $\begin{array}{l}25 \\
(69.4 \%)\end{array}$ & 0.464 & $\begin{array}{l}25 \\
(86.2 \%)\end{array}$ & $\begin{array}{l}12 \\
(70.6 \%)\end{array}$ & 0.182 & $\begin{array}{l}36 \\
(76.6 \%)\end{array}$ & $\begin{array}{l}55 \\
(87.3 \%)\end{array}$ & 0.142 \\
\hline $\begin{array}{l}1,2 \\
3\end{array}$ & $\begin{array}{l}11 \\
(23.4 \%)\end{array}$ & $\begin{array}{l}11 \\
(30.6 \%)\end{array}$ & & $\begin{array}{l}4 \\
(13.8 \%)\end{array}$ & $\begin{array}{l}5 \\
(29.4 \%)\end{array}$ & & $\begin{array}{l}11 \\
(23.4 \%)\end{array}$ & $\begin{array}{l}8 \\
(12.7 \%)\end{array}$ & \\
\hline $\begin{array}{l}\text { Ki67 } \\
<14 \%\end{array}$ & $\begin{array}{l}17 \\
(36.2 \%)\end{array}$ & $\begin{array}{l}8 \\
(22.2 \%)\end{array}$ & 0.170 & $\begin{array}{l}13 \\
(44.8 \%)\end{array}$ & $\begin{array}{l}8 \\
(47.1 \%)\end{array}$ & 0.883 & $\begin{array}{l}17 \\
(36.2 \%)\end{array}$ & $\begin{array}{l}43 \\
(68.3 \%)\end{array}$ & 0.001 \\
\hline$>14 \%$ & $\begin{array}{l}30 \\
(63.8 \%)\end{array}$ & $\begin{array}{l}28 \\
(77.8 \%)\end{array}$ & & $\begin{array}{l}16 \\
(55.2 \%)\end{array}$ & $\begin{array}{l}9 \\
(52.8 \%)\end{array}$ & & $\begin{array}{l}30 \\
(63.8 \%)\end{array}$ & $\begin{array}{l}20 \\
(31.7 \%)\end{array}$ & \\
\hline $\begin{array}{l}\text { Pathological } \\
\text { response }\end{array}$ & $\begin{array}{l}23 \\
(48.9 \%)\end{array}$ & $\begin{array}{l}12 \\
(33.3 \%)\end{array}$ & 0.154 & $\begin{array}{l}15 \\
(51.7 \%)\end{array}$ & $\begin{array}{l}7 \\
(41.2 \%)\end{array}$ & 0.489 & $\begin{array}{l}13 \\
(27.7 \%)\end{array}$ & $\begin{array}{l}9 \\
(14.3 \%)\end{array}$ & 0.083 \\
\hline $\begin{array}{l}\text { pCR } \\
\text { non-pCR }\end{array}$ & $\begin{array}{l}24 \\
(51.1 \%)\end{array}$ & $\begin{array}{l}24 \\
(66.7 \%)\end{array}$ & & $\begin{array}{l}14 \\
(48.3 \%)\end{array}$ & $\begin{array}{l}10 \\
(58.8 \%)\end{array}$ & & $\begin{array}{l}34 \\
(72.3 \%)\end{array}$ & $\begin{array}{l}54 \\
(85.7 \%)\end{array}$ & \\
\hline
\end{tabular}


The DFS period after NAC for the high-FF-TIL group was found to be significantly longer than that for the low-FFTIL group for all cases $(p<0.001$, log-rank) and for all subtypes of TNBC $(p=0.001$, log-rank), HER2BC $(p=$ 0.010 , log-rank), and HRBC ( $p=0.003$, log-rank) (Fig. 3A-D). In comparison of OS between the high-FF-TIL group and the low-FF-TIL group, for all cases ( $p=0.160$, log-rank) and for all subtypes a significant lengthening was not found (TNBC: $p=0.414$, HER2BC: $p=0.888$, HRBC: $p=0.147$, log-rank) (Supplemental Fig. 1A-D). In univariable analysis, for the high-FF-TIL cases, all cases $(p<0.001, H R=0.182)$, and all subtypes of TNBC $(p=$ $0.004, \mathrm{HR}=-0.154), \mathrm{HER} 2 \mathrm{BC}(\mathrm{p}=0.022, \mathrm{HR}=0.136)$, and HRBC $(p=0.007, \mathrm{HR}=0.224)$ contributed to a significant lengthening of the DFS period (Table 3) (Fig. 4A-D). In multivariable analysis as well, high-FF-TIL group classification was an independent factor for recurrence after NAC for all cases $(p<0.001, H R=0.198)$ and all subtypes of TNBC $(p=0.006, H R=0.172), \operatorname{HER} 2 B C(p=0.025, H R=0.135)$, and HRBC $(p=0.007, H R=0.228)$. 
Table 3

Univariable and multivariable analysis with respect to disease-free survival in breast cancer subtypes.

\begin{tabular}{|c|c|c|c|c|c|c|c|}
\hline & & \multicolumn{3}{|c|}{ Univariable analysis } & \multicolumn{3}{|c|}{ Multivariable analysis } \\
\hline \multicolumn{2}{|l|}{ Parameter } & $\begin{array}{l}\text { Hazard } \\
\text { ratio }\end{array}$ & $95 \% \mathrm{Cl}$ & $\begin{array}{l}\mathrm{p} \\
\text { value }\end{array}$ & $\begin{array}{l}\text { Hazard } \\
\text { ratio }\end{array}$ & $95 \% \mathrm{Cl}$ & $\begin{array}{l}p \\
\text { value }\end{array}$ \\
\hline & & \multicolumn{6}{|c|}{ all breast cancers $(n=239)$} \\
\hline Tumor size (cm) & $\leq 2$ vs $>2$ & 1.594 & $\begin{array}{l}0.631- \\
4.030\end{array}$ & 0.324 & & & \\
\hline $\begin{array}{l}\text { Lymph node } \\
\text { status }\end{array}$ & $\begin{array}{l}\text { Negative vs } \\
\text { Positive }\end{array}$ & 2.2494 & $\begin{array}{l}1.058- \\
5.879\end{array}$ & 0.037 & 2.286 & $\begin{array}{l}0.969- \\
5.393\end{array}$ & 0.059 \\
\hline Nuclear grade & $1-2$ vs 3 & 1.126 & $\begin{array}{l}0.573- \\
2.214\end{array}$ & 0.730 & & & \\
\hline Ki67 (\%) & $\leq 14$ vs $>14$ & 0.717 & $\begin{array}{l}0.403- \\
1.277\end{array}$ & 0.259 & & & \\
\hline $\begin{array}{l}\text { Pathological } \\
\text { response }\end{array}$ & $\begin{array}{l}\text { pCR vs non- } \\
\text { pCR }\end{array}$ & 0.561 & $\begin{array}{l}0.291- \\
1.083\end{array}$ & 0.085 & 0.783 & $\begin{array}{l}0.401- \\
1.529\end{array}$ & 0.474 \\
\hline \multirow[t]{2}{*}{ FF-TILs } & High vs Low & 0.182 & $\begin{array}{l}0.088- \\
0.379\end{array}$ & $\begin{array}{l}< \\
0.001\end{array}$ & 0.198 & $\begin{array}{l}0.094- \\
0.418\end{array}$ & $\begin{array}{l}< \\
0.001\end{array}$ \\
\hline & & \multicolumn{3}{|c|}{$\operatorname{TNBC}(\mathrm{n}=83)$} & & & \\
\hline Tumor size (cm) & $\leq 2$ vs $>2$ & 0.701 & $\begin{array}{l}0.200- \\
2.464\end{array}$ & 0.580 & & & \\
\hline $\begin{array}{l}\text { Lymph node } \\
\text { status }\end{array}$ & $\begin{array}{l}\text { Negative vs } \\
\text { Positive }\end{array}$ & 1.059 & $\begin{array}{l}0.340- \\
3.293\end{array}$ & 0.921 & & & \\
\hline Nuclear grade & $1-2$ vs 3 & 0.934 & $\begin{array}{l}0.253- \\
3.453\end{array}$ & 0.919 & & & \\
\hline Ki67 (\%) & $\leq 14$ vs $>14$ & 0.961 & $\begin{array}{l}0.334- \\
2.765\end{array}$ & 0.941 & & & \\
\hline $\begin{array}{l}\text { Pathological } \\
\text { response }\end{array}$ & $\begin{array}{l}\text { pCR vs non- } \\
\text { pCR }\end{array}$ & 0.380 & $\begin{array}{l}0.122- \\
1.182\end{array}$ & 0.095 & 0.492 & $\begin{array}{l}0.157- \\
1.545\end{array}$ & 0.225 \\
\hline \multirow[t]{2}{*}{ FF-TILs } & High vs Low & 0.154 & $\begin{array}{l}0.044- \\
0.543\end{array}$ & 0.004 & 0.172 & $\begin{array}{l}0.048- \\
0.609\end{array}$ & 0.006 \\
\hline & & \multicolumn{3}{|c|}{ HER2BC $(n=46)$} & & & \\
\hline Tumor size (cm) & $\leq 2$ vs $>2$ & 0.176 & $\begin{array}{l}0.220- \\
14.649\end{array}$ & 0.584 & & & \\
\hline $\begin{array}{l}\text { Lymph node } \\
\text { status }\end{array}$ & $\begin{array}{l}\text { Negative vs } \\
\text { Positive }\end{array}$ & 3.201 & $\begin{array}{l}0.393- \\
26.099\end{array}$ & 0.277 & & & \\
\hline Nuclear grade & $1-2$ vs 3 & 0.452 & $\begin{array}{l}0.055- \\
3.688\end{array}$ & 0.458 & & & \\
\hline
\end{tabular}




\begin{tabular}{|c|c|c|c|c|c|c|c|}
\hline \multirow[b]{2}{*}{ Ki67 (\%) } & \multirow[b]{2}{*}{$\leq 14$ vs $>14$} & \multicolumn{3}{|c|}{ Univariable analysis } & \multicolumn{3}{|c|}{ Multivariable analysis } \\
\hline & & 0.377 & $\begin{array}{l}0.088- \\
1.619\end{array}$ & 0.190 & 0.391 & $\begin{array}{l}0.089- \\
1.721\end{array}$ & 0.214 \\
\hline $\begin{array}{l}\text { Pathological } \\
\text { response }\end{array}$ & $\begin{array}{l}\text { pCR vs non- } \\
\text { pCR }\end{array}$ & 0.572 & $\begin{array}{l}0.136- \\
2.403\end{array}$ & 0.445 & & & \\
\hline \multirow[t]{2}{*}{ FF-TILs } & High vs Low & 0.136 & $\begin{array}{l}0.025- \\
0.748\end{array}$ & 0.022 & 0.135 & $\begin{array}{l}0.024- \\
0.775\end{array}$ & 0.025 \\
\hline & & \multicolumn{3}{|c|}{$\operatorname{HRBC}(n=110)$} & & & \\
\hline Tumor size (cm) & $\leq 2$ vs $>2$ & 4.418 & $\begin{array}{l}0.595- \\
32.821\end{array}$ & 0.146 & & & \\
\hline $\begin{array}{l}\text { Lymph node } \\
\text { status }\end{array}$ & $\begin{array}{l}\text { Negative vs } \\
\text { Positive }\end{array}$ & 7.711 & $\begin{array}{l}1.039- \\
57.236\end{array}$ & 0.046 & 7.592 & $\begin{array}{l}1.022- \\
56.411\end{array}$ & 0.048 \\
\hline Nuclear grade & $1-2$ vs 3 & 1.234 & $\begin{array}{l}0.456- \\
3.341\end{array}$ & 0.679 & & & \\
\hline Ki67 (\%) & $\leq 14$ vs $>14$ & 0.703 & $\begin{array}{l}0.304- \\
1.624\end{array}$ & 0.410 & & & \\
\hline $\begin{array}{l}\text { Pathological } \\
\text { response }\end{array}$ & $\begin{array}{l}\text { pCR vs non- } \\
\text { pCR }\end{array}$ & 0.816 & $\begin{array}{l}0.301- \\
2.210\end{array}$ & 0.689 & & & \\
\hline FF-TILs & High vs Low & 0.224 & $\begin{array}{l}0.076- \\
0.663\end{array}$ & 0.007 & 0.228 & $\begin{array}{l}0.077- \\
0.672\end{array}$ & 0.007 \\
\hline
\end{tabular}

When receiver operating characteristic (ROC) analysis was performed, for all breast cancer cases, VS (rea under the curve [AUC]: 0.701) was shown to have better results than TS (AUC: 0.582) (Fig. 5A). Similar results were also found with investigation based on subtype: TNBC (AUC: TS $=0.616, V S=0.735$ ), HER2BC (AUC: TS $=0.618$, VS $=0.730)$, and HRBC (AUC: TS 0.530, VS = 0.660) (Fig. 5B-D).

\section{Discussion}

Recently, in many large-scale prospective clinical trials, TILs have been proven to be useful as a prognostic factor [4] [6, 8, 9, 31]. These reports have suggested that TILs can be useful as clinical biomarkers, and today attempts to unify their evaluation methods are underway [3, 4]. According to the International Working Group, in the evaluation of TILs, the percentage of immunocytes within a tumor stroma should be measured at the border of the cancer infiltration region [3]. However, there is not yet a standardized opinion regarding determination of the region within the tumor stroma. Therefore, we also paid attention to FF in regions that were within the tumor stroma and were surrounded by infiltrating tumor cells. Wide-ranging FF contribute to the tumor's acquisition of a malignant condition due to the hypoxic environment within the tumor, and are related to prognosis and drug resistance [32-34]. In the present study, when we investigated TILs that exist within FF as FF-TILs in patients undergoing NAC, the high-FF-TIL group had a longer DFS period after NAC than did the low-FF-TIL group. 
When studied by subtype, TILs have been considered useful as prediction markers for treatment effect in subtypes with high immunological action, such as TNBC and HER2BC $[4,8,9,31]$. However, in HRBC, which has the highest frequency, there are few reports that show a clinical relationship with TILs. In the present study, FFTILs have been proven useful as prognostic factors following NAC not only for TNBC and HER2BC, but also for HRBC. Furthermore, for non-HRBC, such as TNBC and HER2BC, it is thought that wide-ranging FF exist [34], and in the present study as well, for non-HRBC, we observed that FF-TILs were significantly high.

In the current study, a reason that outcome prediction was possible even for HRBC is the heterogeneity of TILs in the cancer microenvironment. ER, which is important for the occurrence and development of HRBC, is activated not just by estrogen but also by the signal cascade of a pathway via phosphorylation due to various growth factors, and the control of ER depends on the cancer microenvironment [35]. However, there are reports that, in a hypoxic environment, expression of hormone receptors is weakened [36,37]. That is, it is thought that, in an FF region, hormone receptors are weakened owing to the hypoxic environment; therefore, biomarkers for ERnegative cancers are useful. It is possible to dynamically understand the changes in the cancer microenvironment more precisely with FF-TILs than with the previous evaluation range of TILs; therefore, we believe it may be a better evaluation method.

In the present study, patients in the low-FF-TIL group experienced a high recurrence rate, and perhaps, depending on the subtype, adjuvant therapy should have been added. That is, in the selection of post-surgical adjuvant therapy for patients undergoing NAC, it is suggested that determination using FF-TILs can contribute to the choice of a proper treatment strategy.

However, the retrospective nature of this study is a limitation, and regarding adjuvant therapy after NAC, since there are differences among cases, and since it is difficult to evaluate FF with CNB specimens, it is necessary to obtain more tissue through the use of methods such as VAB during diagnoses. In the previous report, we classified FF long diameter $8 \mathrm{~mm}$ as positive / negative as cutoff. However, with this method, evaluable FFs are limited. In this study, we used FF as the convergent focal point of the fiber component surrounded by infiltrating tumor cells without using the cutoff value. That is, it was possible to evaluate FF even if the area was small. Therefore, the evaluation rate of FF is higher than the previous report. Even a minute specimen at CNB or VAB, in the method we used. Evaluation of FF becomes possible.

\section{Conclusions}

It is suggested that FF-TILs are a useful factor for predicting recurrence of breast cancer after NAC, and they may be a more precise indicator than previous evaluation methods with TILs.

\section{Abbreviations}

NAC: neoadjuvant chemotherapy, TILs: tumor-infiltrating lymphocytes, FF: fibrotic foci, UICC: Union for International Cancer Control, CNB: core needle biopsy, VAB: vacuum-assisted biopsy, CT: computed tomography, US: ultrasonography, ER: estrogen receptor, PgR: progesterone receptor, HER: human epidermal growth factor receptor, HER2BC: HER2-enriched, TNBC: triple-negative breast cancer, HRBC: hormone receptor-positive breast cancer, RECIST: Response Evaluation Criteria in Solid Tumors, pCR: pathological complete response, OS: overall survival, DFS: disease-free survival, REMARK: Reporting Recommendations for Tumor Marker prognostic

Page $13 / 22$ 
Studies, HE: hematoxylin and eosin, TS: Training Set, VS: Validation Set, HR: hazard ratio, Cl: confidence interval, ROC: receiver operating characteristic, AUC: area under the curve.

\section{Declarations}

\section{Ethics approval and consent to participate}

Written informed consent was obtained from all subjects. This research conformed to the provisions of the Declaration of Helsinki in 2013. All patients were informed of the investigational nature of this study and provided their written, informed consent. The study protocol was approved by the Ethics Committee of Osaka City University (\#926).

\section{Consent for publication}

Not applicable.

\section{Availability of data and materials}

The data and materials used and analyzed in the current study would be available from the corresponding author on request.

\section{Competing interests}

The authors declare that they have no competing interests.

\section{Funding}

This study was supported in part by Grants-in Aid for Scientific Research (KAKENHI, Nos. 25461992, 20K08938, and 17K10559) from the Ministry of Education, Science, Sports, Culture and Technology of Japan.

\section{Authors' contributions}

All authors were involved in the preparation of this manuscript. YA collected the data, and wrote the manuscript. SK, RK, AY, KT, SI, WG and TM performed the operation and designed the study. YA, SK, MS and HT summarized the data and revised the manuscript. $\mathrm{KH}$ and $\mathrm{MO}$ substantial contribution to the study design, performed the operation, and revised the manuscript. All authors read and approved the final manuscript.

\section{Acknowledgements}

The authors would like to thank Yuko Kuwae from the Department of Diagnostic Pathology, Osaka City University Graduate School of Medicine, for providing assistance with immunohistochemical scoring. And, we thank Yayoi Matsukiyo and Tomomi Okawa (Department of Breast and Endocrine Surgery, Osaka City University Graduate School of Medicine) for helpful advice regarding data management.

\section{Author's information}

${ }^{1}$ Department of Breast and Endocrine Surgery, Osaka City University Graduate School of Medicine, 1-4-3 Asahimachi, Abeno-ku, Osaka 545-8585, Japan. 
${ }^{2}$ Department of Gastrointestinal Surgery, Osaka City University Graduate School of Medicine, 1-4-3 Asahi-machi, Abeno-ku, Osaka 545-8585, Japan.

\section{References}

1. Hanahan D, Weinberg RA. Hallmarks of cancer: the next generation. Cell. 2011;144(5):646-74.

2. Couzin-Frankel J. Breakthrough of the year 2013. Cancer immunotherapy. Science. 2013;342(6165):14323.

3. Salgado R, Denkert C, Demaria S, Sirtaine N, Klauschen F, Pruneri G, Wienert S, Van den Eynden G, Baehner FL, Penault-Llorca F, et al. The evaluation of tumor-infiltrating lymphocytes (TILs) in breast cancer: recommendations by an International TILs Working Group 2014. Ann Oncol. 2015;26(2):259-71.

4. Savas P, Salgado R, Denkert C, Sotiriou C, Darcy PK, Smyth MJ, Loi S. Clinical relevance of host immunity in breast cancer: from TILs to the clinic. Nat Rev Clin Oncol. 2016;13(4):228-41.

5. Asano Y, Kashiwagi S, Goto W, Kurata K, Noda S, Takashima T, Onoda N, Tanaka S, Ohsawa M, Hirakawa K. Tumour-infiltrating CD8 to FOXP3 lymphocyte ratio in predicting treatment responses to neoadjuvant chemotherapy of aggressive breast cancer. Br J Surg. 2016;103(7):845-54.

6. Adams S, Gray RJ, Demaria S, Goldstein L, Perez EA, Shulman LN, Martino S, Wang M, Jones VE, Saphner TJ, et al. Prognostic value of tumor-infiltrating lymphocytes in triple-negative breast cancers from two phase III randomized adjuvant breast cancer trials: ECOG 2197 and ECOG 1199. J Clin Oncol. 2014;32(27):295966.

7. Denkert C, Loibl S, Noske A, Roller M, Muller BM, Komor M, Budczies J, Darb-Esfahani S, Kronenwett R, Hanusch $\mathrm{C}$, et al. Tumor-associated lymphocytes as an independent predictor of response to neoadjuvant chemotherapy in breast cancer. J Clin Oncol. 2010;28(1):105-13.

8. Loi S. Host antitumor immunity plays a role in the survival of patients with newly diagnosed triple-negative breast cancer. J Clin Oncol. 2014;32(27):2935-7.

9. Loi S, Sirtaine N, Piette F, Salgado R, Viale G, Van Eenoo F, Rouas G, Francis P, Crown JP, Hitre E, et al. Prognostic and predictive value of tumor-infiltrating lymphocytes in a phase III randomized adjuvant breast cancer trial in node-positive breast cancer comparing the addition of docetaxel to doxorubicin with doxorubicin-based chemotherapy: BIG 02-98. J Clin Oncol. 2013;31(7):860-7.

10. West NR, Milne K, Truong PT, Macpherson N, Nelson BH, Watson PH. Tumor-infiltrating lymphocytes predict response to anthracycline-based chemotherapy in estrogen receptor-negative breast cancer. Breast Cancer Res. 2011;13(6):R126.

11. Ono M, Tsuda H, Shimizu C, Yamamoto S, Shibata T, Yamamoto H, Hirata T, Yonemori K, Ando M, Tamura K, et al. Tumor-infiltrating lymphocytes are correlated with response to neoadjuvant chemotherapy in triplenegative breast cancer. Breast Cancer Res Treat. 2012;132(3):793-805.

12. True LD, Zhang H, Ye M, Huang CY, Nelson PS, von Haller PD, Tjoelker LW, Kim JS, Qian WJ, Smith RD, et al. CD90/THY1 is overexpressed in prostate cancer-associated fibroblasts and could serve as a cancer biomarker. Mod Pathol. 2010;23(10):1346-56.

13. Sandoval P, Jimenez-Heffernan JA, Rynne-Vidal A, Perez-Lozano ML, Gilsanz A, Ruiz-Carpio V, Reyes R, Garcia-Bordas J, Stamatakis K, Dotor J, et al. Carcinoma-associated fibroblasts derive from mesothelial cells via mesothelial-to-mesenchymal transition in peritoneal metastasis. J Pathol. 2013;231(4):517-31. 
14. Tsuyada A, Chow A, Wu J, Somlo G, Chu P, Loera S, Luu T, Li AX, Wu X, Ye W, et al. CCL2 mediates cross-talk between cancer cells and stromal fibroblasts that regulates breast cancer stem cells. Cancer Res. 2012;72(11):2768-79.

15. Hasebe T, Tsuda H, Hirohashi S, Shimosato Y, Iwai M, Imoto S, Mukai K. Fibrotic focus in invasive ductal carcinoma: an indicator of high tumor aggressiveness. Jpn J Cancer Res. 1996;87(4):385-94.

16. Hasebe T, Tsuda H, Hirohashi S, Shimosato Y, Tsubono Y, Yamamoto H, Mukai K. Fibrotic focus in infiltrating ductal carcinoma of the breast: a significant histopathological prognostic parameter for predicting the long-term survival of the patients. Breast Cancer Res Treat. 1998;49(3):195-208.

17. Maiorano E, Regan MM, Viale G, Mastropasqua MG, Colleoni M, Castiglione-Gertsch M, Price KN, Gelber RD, Goldhirsch A, Coates AS. Prognostic and predictive impact of central necrosis and fibrosis in early breast cancer: results from two International Breast Cancer Study Group randomized trials of chemoendocrine adjuvant therapy. Breast Cancer Res Treat. 2010;121(1):211-8.

18. Mujtaba SS, Ni YB, Tsang JY, Chan SK, Yamaguchi R, Tanaka M, Tan PH, Tse GM. Fibrotic focus in breast carcinomas: relationship with prognostic parameters and biomarkers. Ann Surg Oncol. 2013;20(9):2842-9.

19. Colpaert C, Vermeulen P, van Beest P, Goovaerts G, Weyler J, Van Dam P, Dirix L, Van Marck E. Intratumoral hypoxia resulting in the presence of a fibrotic focus is an independent predictor of early distant relapse in lymph node-negative breast cancer patients. Histopathology. 2001;39(4):416-25.

20. Van den Eynden GG, Colpaert CG, Couvelard A, Pezzella F, Dirix LY, Vermeulen PB, Van Marck EA, Hasebe T. A fibrotic focus is a prognostic factor and a surrogate marker for hypoxia and (lymph)angiogenesis in breast cancer: review of the literature and proposal on the criteria of evaluation. Histopathology. 2007;51(4):440-51.

21. Greene FL, Sobin LH. A worldwide approach to the TNM staging system: collaborative efforts of the AJCC and UICC. J Surg Oncol. 2009;99(5):269-72.

22. Goldhirsch A, Wood WC, Coates AS, Gelber RD, Thurlimann B, Senn HJ. Panel m: Strategies for subtypesdealing with the diversity of breast cancer: highlights of the St. Gallen International Expert Consensus on the Primary Therapy of Early Breast Cancer 2011. Ann Oncol. 2011;22(8):1736-47.

23. Mauri D, Pavlidis N, loannidis JP. Neoadjuvant versus adjuvant systemic treatment in breast cancer: a metaanalysis. J Natl Cancer Inst. 2005;97(3):188-94.

24. Mieog JS, van der Hage JA, van de Velde CJ. Preoperative chemotherapy for women with operable breast cancer. Cochrane Database Syst Rev 2007(2):CD005002.

25. Kawajiri H, Takashima T, Onoda N, Kashiwagi S, Noda S, Ishikawa T, Wakasa K, Hirakawa K. Efficacy and feasibility of neoadjuvant chemotherapy with FEC 100 followed by weekly paclitaxel for operable breast cancer. Oncol Lett. 2012;4(4):612-6.

26. Eisenhauer EA, Therasse P, Bogaerts J, Schwartz LH, Sargent D, Ford R, Dancey J, Arbuck S, Gwyther S, Mooney $\mathrm{M}$, et al. New response evaluation criteria in solid tumours: revised RECIST guideline (version 1.1). Eur J Cancer. 2009;45(2):228-47.

27. Kashiwagi S, Onoda N, Asano Y, Kurata K, Morisaki T, Noda S, Kawajiri H, Takashima T, Hirakawa K. Partial mastectomy using manual blunt dissection (MBD) in early breast cancer. BMC Surg. 2015;15:117.

28. Wolmark N, Wang J, Mamounas E, Bryant J, Fisher B. Preoperative chemotherapy in patients with operable breast cancer: nine-year results from National Surgical Adjuvant Breast and Bowel Project B-18. J Nat/ 
Cancer Inst Monogr 2001(30):96-102.

29. McShane LM, Altman DG, Sauerbrei W, Taube SE, Gion M, Clark GM. Statistics Subcommittee of the NCIEWGoCD: Reporting recommendations for tumor marker prognostic studies. J Clin Oncol. 2005;23(36):9067-72.

30. Mao Y, Qu Q, Zhang Y, Liu J, Chen X, Shen K: The value of tumor infiltrating lymphocytes (TILs) for predicting response to neoadjuvant chemotherapy in breast cancer: a systematic review and meta-analysis. PLoS One 2014, 9(12):e115103.

31. Denkert C, von Minckwitz G, Brase JC, Sinn BV, Gade S, Kronenwett R, Pfitzner BM, Salat C, Loi S, Schmitt WD, et al. Tumor-infiltrating lymphocytes and response to neoadjuvant chemotherapy with or without carboplatin in human epidermal growth factor receptor 2-positive and triple-negative primary breast cancers. J Clin Oncol. 2015;33(9):983-91.

32. Colpaert CG, Vermeulen PB, Fox SB, Harris AL, Dirix LY, Van Marck EA. The presence of a fibrotic focus in invasive breast carcinoma correlates with the expression of carbonic anhydrase IX and is a marker of hypoxia and poor prognosis. Breast Cancer Res Treat. 2003;81(2):137-47.

33. Kornegoor R, Verschuur-Maes AH, Buerger H, Hogenes MC, de Bruin PC, Oudejans JJ, Hinrichs B, van Diest PJ. Fibrotic focus and hypoxia in male breast cancer. Mod Pathol. 2012;25(10):1397-404.

34. Van den Eynden GG, Smid M, Van Laere SJ, Colpaert CG, Van der Auwera I, Bich TX, van Dam P, den Bakker MA, Dirix LY, Van Marck EA, et al. Gene expression profiles associated with the presence of a fibrotic focus and the growth pattern in lymph node-negative breast cancer. Clin Cancer Res. 2008;14(10):2944-52.

35. Omoto Y, Eguchi H, Yamamoto-Yamaguchi Y, Hayashi S. Estrogen receptor (ER) beta1 and ERbetacx/beta2 inhibit ERalpha function differently in breast cancer cell line MCF7. Oncogene. 2003;22(32):5011-20.

36. Yi JM, Kwon HY, Cho JY, Lee YJ. Estrogen and hypoxia regulate estrogen receptor alpha in a synergistic manner. Biochem Biophys Res Commun. 2009;378(4):842-6.

37. Wolff M, Kosyna FK, Dunst J, Jelkmann W, Depping R. Impact of hypoxia inducible factors on estrogen receptor expression in breast cancer cells. Arch Biochem Biophys. 2017;613:23-30.

\section{Figures}


Fig. 1 Asano Y. et al.

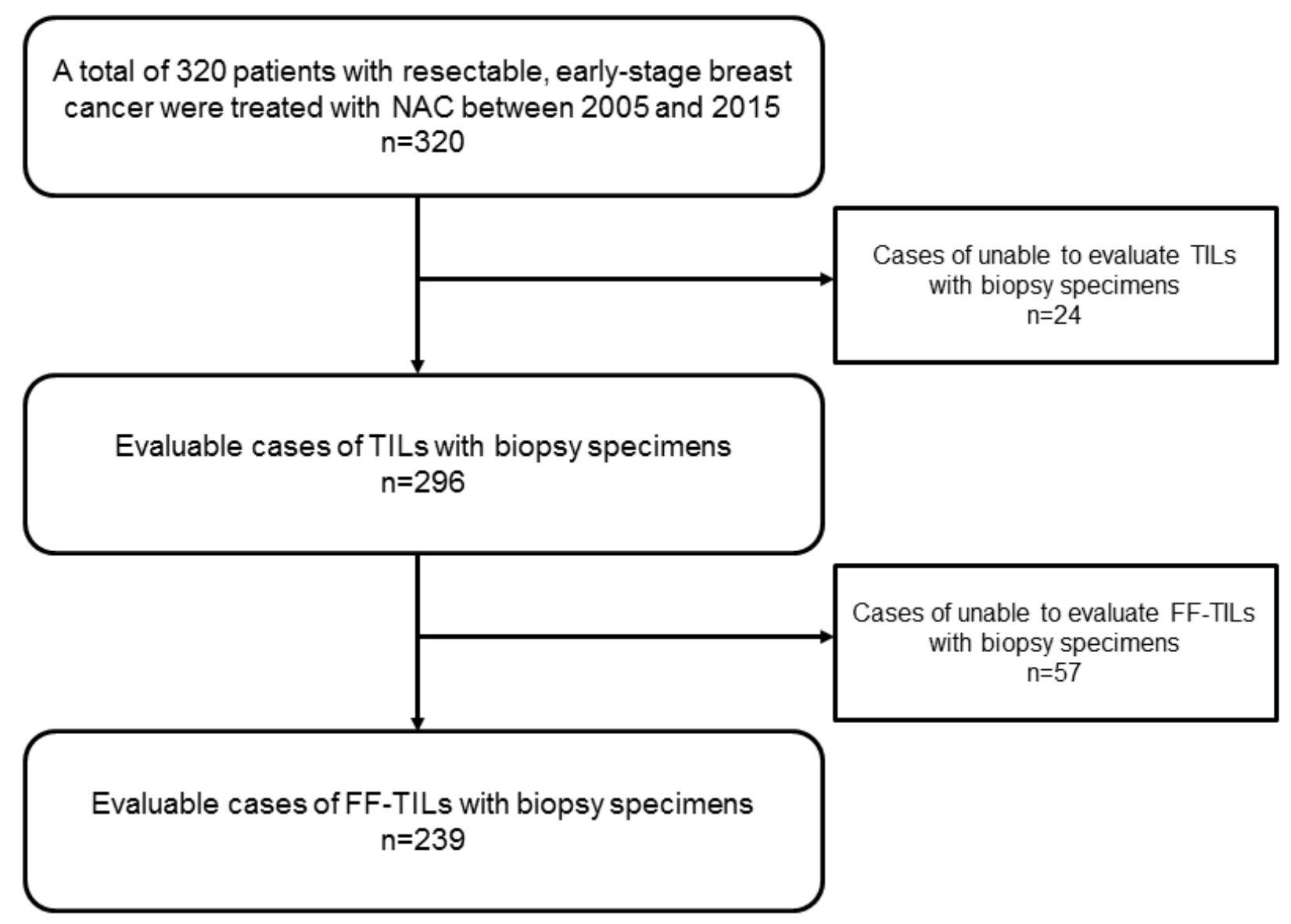

Figure 1

Consort diagram. The present study included 239 participants, following the exclusion of 24 patients in whom the evaluation of TILs from the biopsy samples was difficult, and another 57 patients in whom the evaluation of FF-TILs was difficult. 


\section{Fig. 2 Asano Y. et al.}

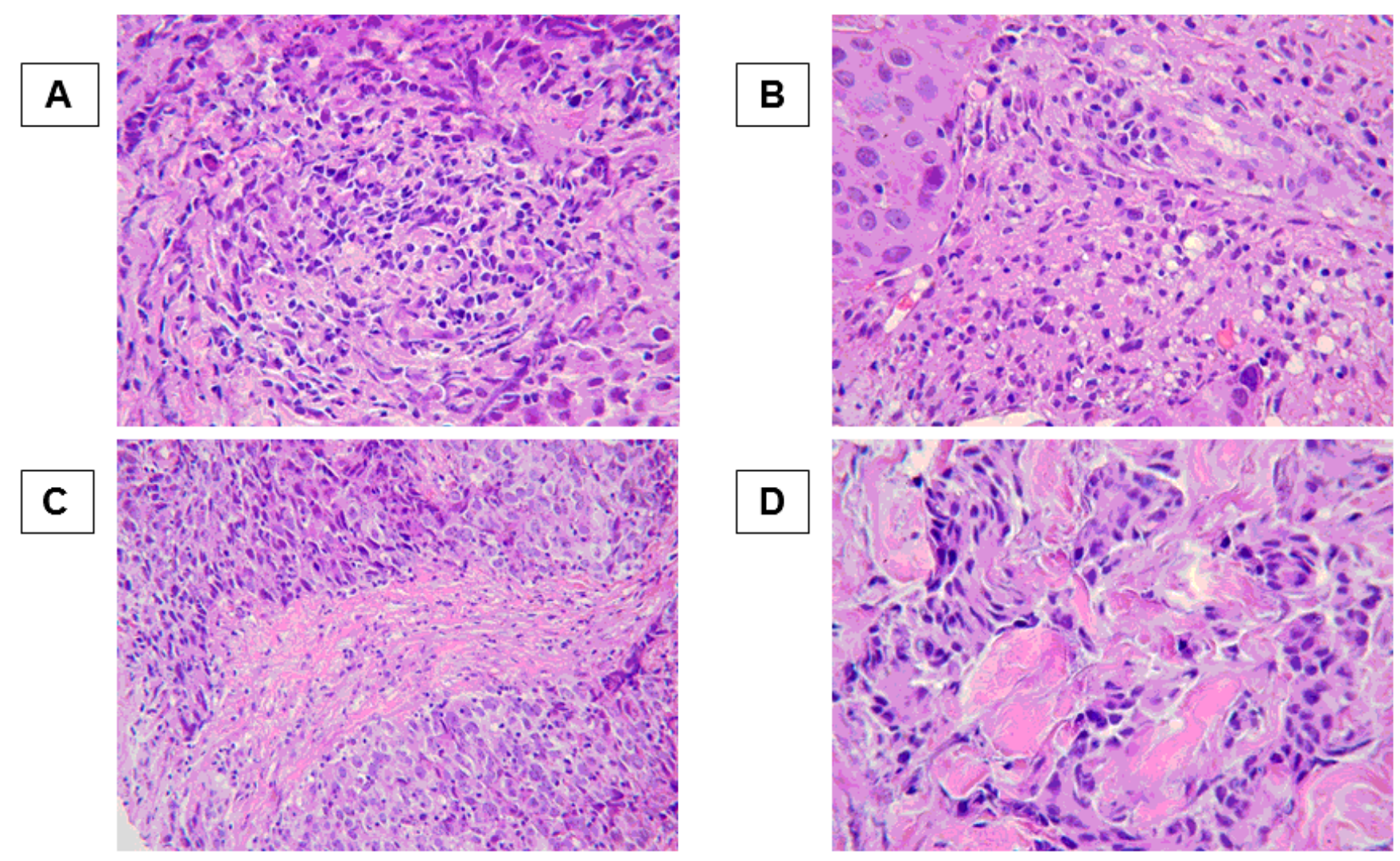

Figure 2

Histopathological evaluation of FF-TILs. Histopathologic analysis of the percentage of FF-TILs was performed on a single full-face hematoxylin and eosin-stained tumor section. FF-TILs were defined as the percentage of tumor stroma containing infiltrating lymphocytes. Proportional scores were defined as 3, 2, 1, and 0 if the area of stroma with lymphoplasmacytic infiltration around invasive tumor cell nests was > 50\% (A); > $10-50 \%(B)$; $\leq$ 10\% (C); and absent (D), respectively. 


\section{Fig. 3 Asano Y. et al.}

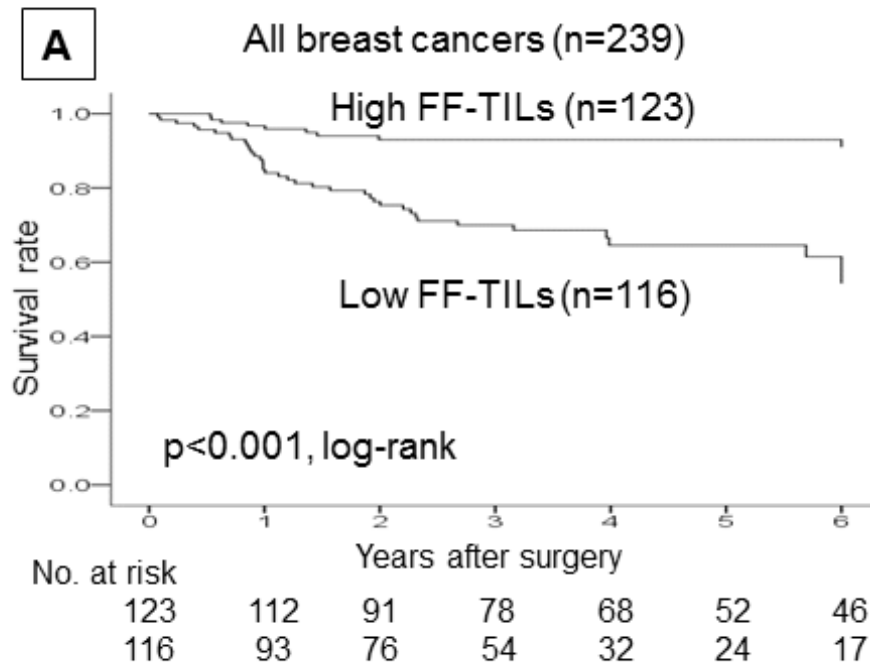

B Triple-negative breast cancers $(n=83)$

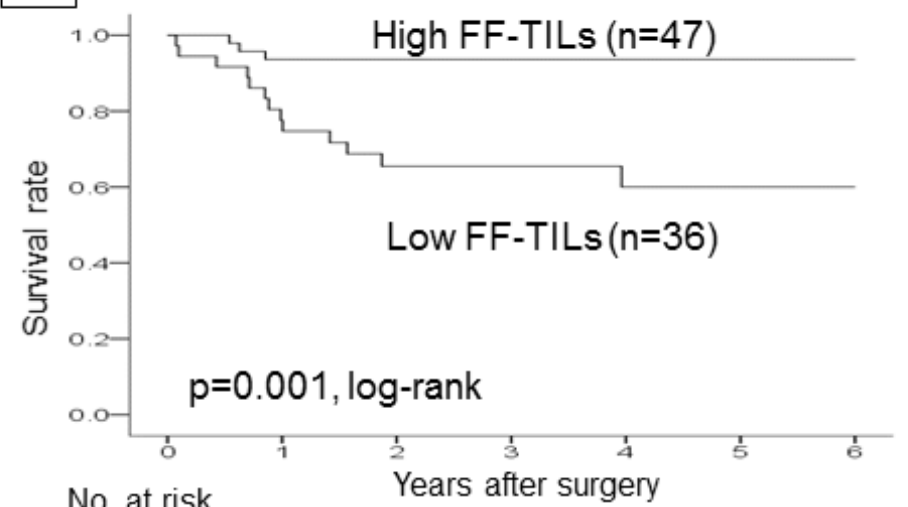

No. at risk

$\begin{array}{lllllll}47 & 43 & 35 & 30 & 24 & 18 & 16\end{array}$

C HER2-enriched breast cancers $(n=46)$

D Hormone receptor-positive breast cancer $(n=110)$
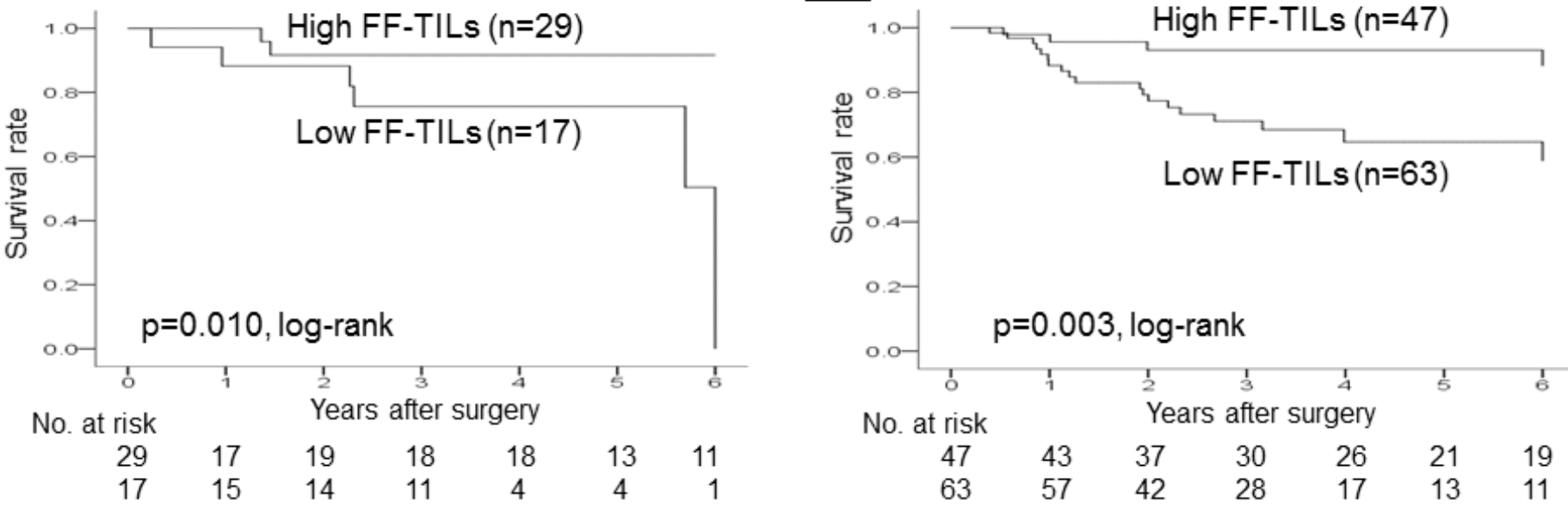

Figure 3

The disease-free survival (DFS) period after NAC. The DFS period after NAC for the high-FF-TIL group was found to be significantly longer than that for the low-FF-TIL group for all cases ( $p<0.001$, log-rank) $(A)$ and for all subtypes of TNBC ( $p=0.001$, log-rank) (B), HER2BC ( $p=0.010$, log-rank $)(C)$, and HRBC $(p=0.003$, log-rank $)(D)$. 


\section{Fig. 4 Asano Y. et al.}

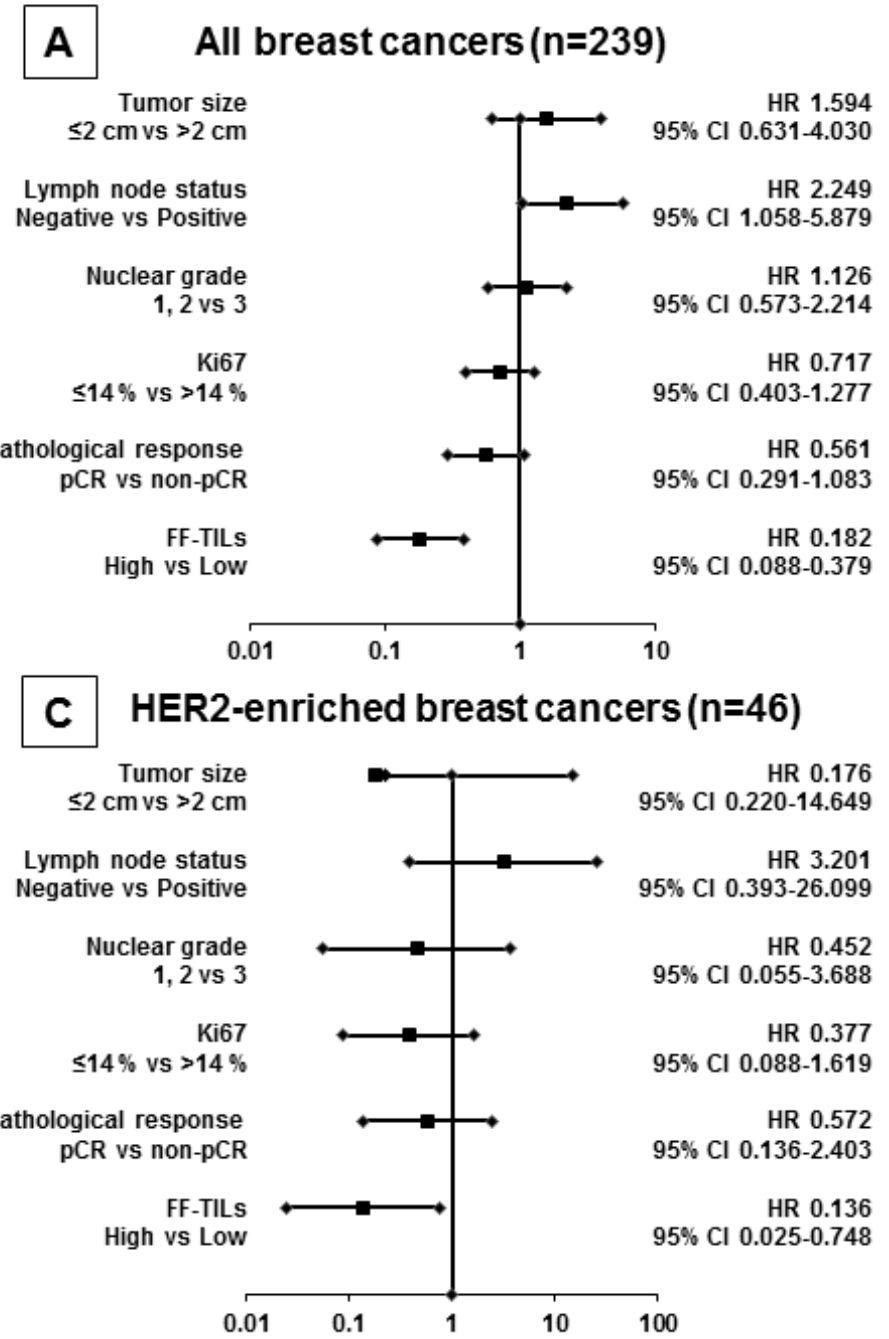

B Triple-negative breast cancers $(\mathbf{n}=\mathbf{8 1})$

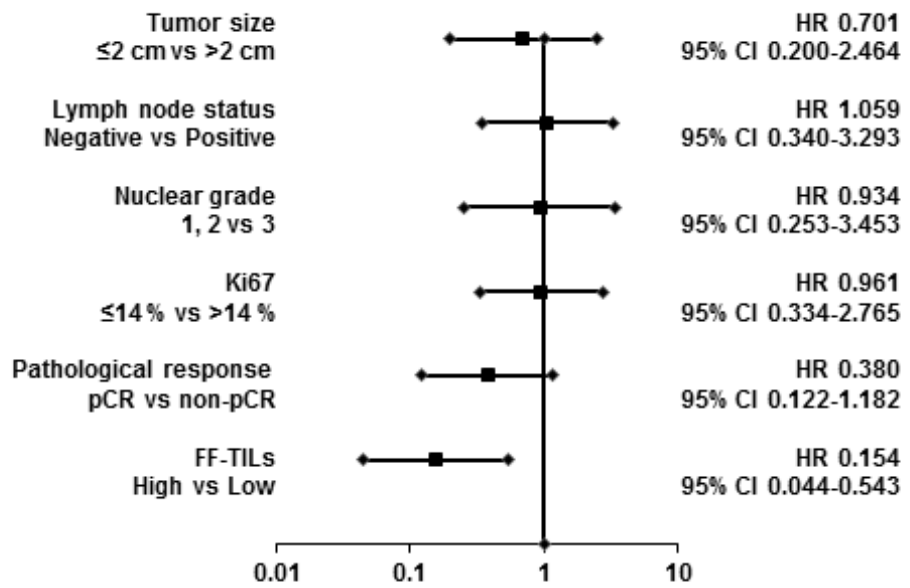

D Hormone receptor-positive breast cancer $(n=110)$

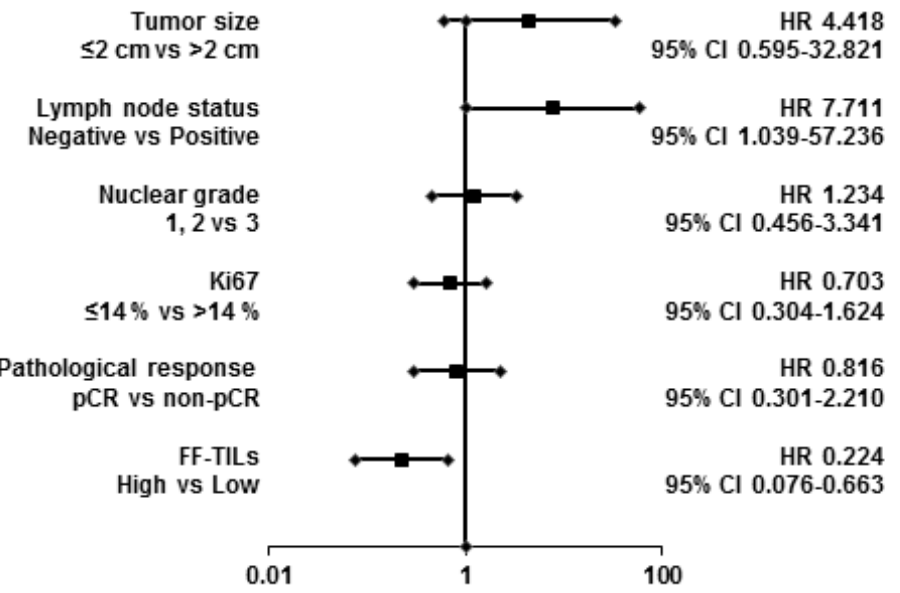

Figure 4

Forrest plot. In univariable analysis, for the high-FF-TIL cases, all cases $(p<0.001, H R=0.182)$, and all subtypes of TNBC ( $p=0.004, \mathrm{HR}=-0.154), \operatorname{HER} 2 B C(p=0.022, \mathrm{HR}=0.136)$, and HRBC ( $=0.007, \mathrm{HR}=0.224)$ contributed to a significant lengthening of the DFS period. 


\section{Fig. 5 Asano Y. et al.}
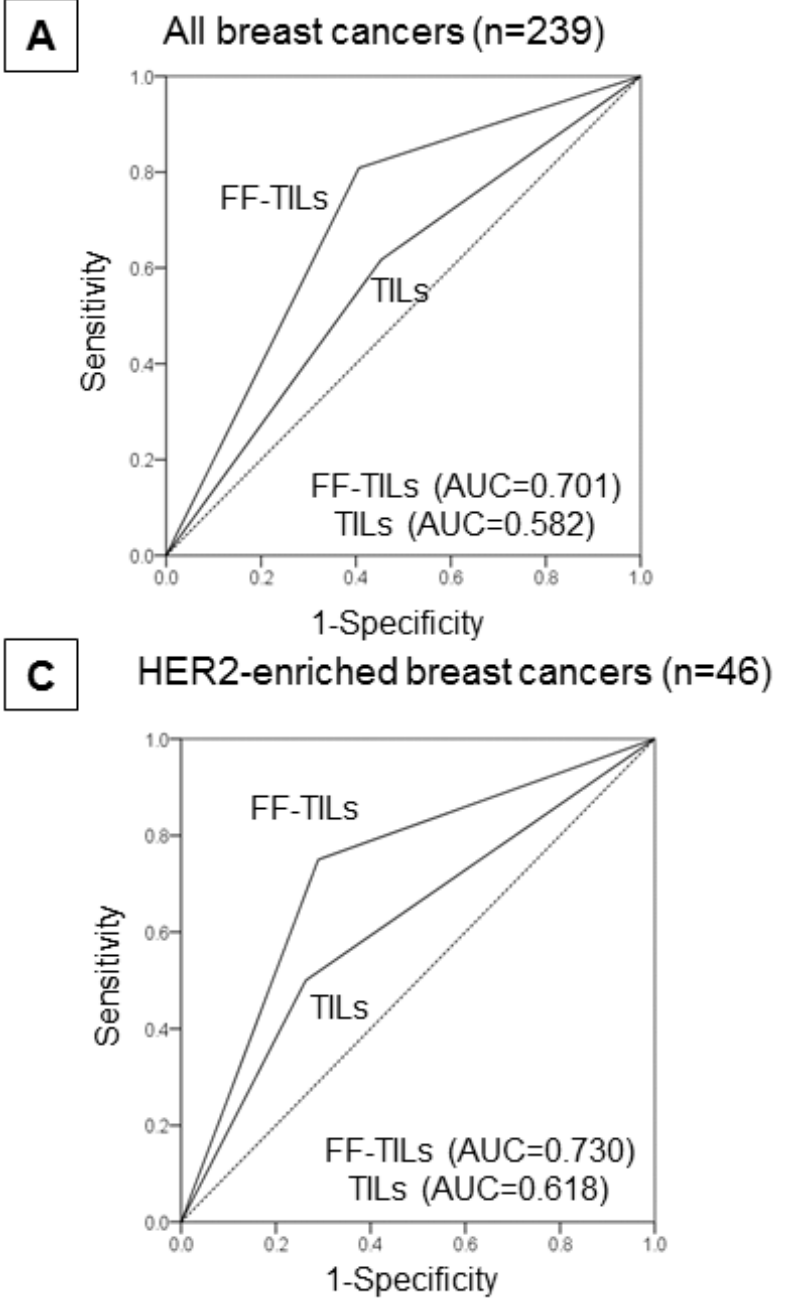

B Triple-negative breast cancers $(n=83)$

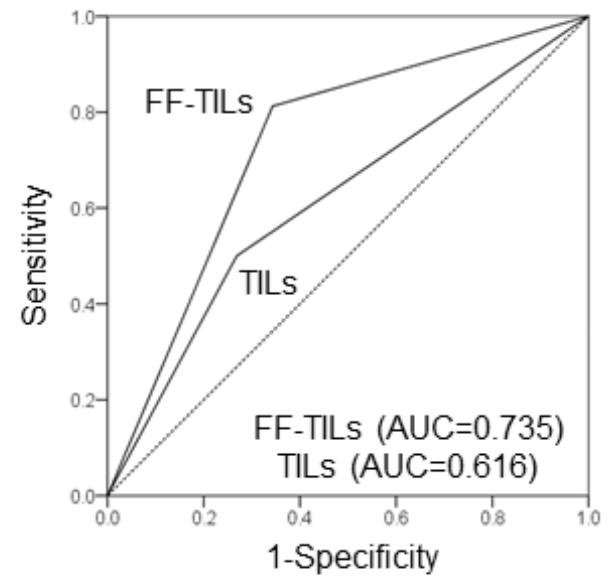

D Hormone receptor-positive breast cancer $(n=110)$

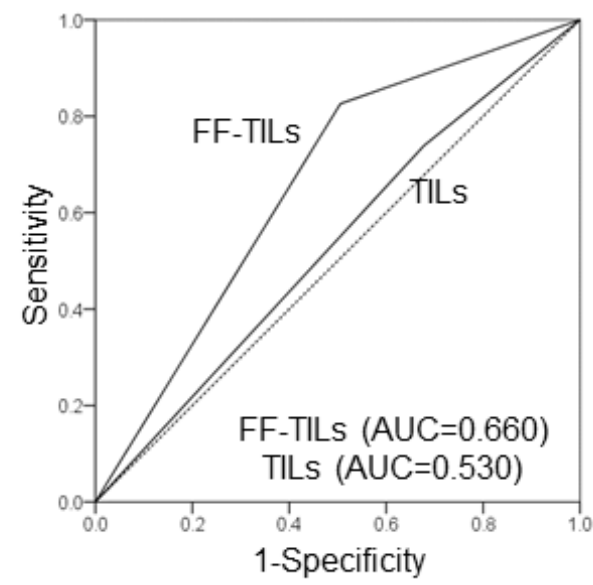

\section{Figure 5}

Receiver operating characteristic (ROC) analysis. When ROC analysis was performed, for all breast cancer cases, VS (rea under the curve [AUC]: 0.701) was shown to have better results than TS (AUC: 0.582) (A). Similar results were also found with investigation based on subtype: TNBC (AUC: TS $=0.616, \mathrm{VS}=0.735)(B)$, HER2BC (AUC: $\mathrm{TS}=0.618, \mathrm{VS}=0.730)(\mathrm{C})$, and HRBC (AUC: TS 0.530, VS =0.660) (D).

\section{Supplementary Files}

This is a list of supplementary files associated with this preprint. Click to download.

- SupplementalFig.1.tif 